\title{
Design of One Line Bus Monitoring System Mine Stratum Freezing Based on Database
}

\author{
LIU Xin-jun ${ }^{1 a}$, WU Dong-ling ${ }^{2 b}$ \\ ${ }^{1}$ Department of Computer Project, Tangshan Vocational College of Science and Technology, \\ Tangshan Hebei 063000, P.R.China; \\ ${ }^{2}$ Department of Information Project, Tangshan Vocational \& Technical College, Tangshan Hebei \\ 063000, P.R.China
}

Keywords: ground freezing; one line bus; monitoring system; reliability

\begin{abstract}
The observation of the frozen wall cannot be directly monitored, and it is needed to take an effective monitoring system to monitor. This paper presents the application of the one line bus technology to develop the mine ground freezing monitoring system, and the system is introduced in detail. By combining with examples, it is proved that this system has high reliability and stability, but it also needs to improve the performance of prediction.
\end{abstract}

\section{Introduction}

In coal mining, a large number of freezing pipes are laying out. In the inside of these freezing pipes, the circulating cooling medium is loaded, as a result of the cooling fluid can take away the heat around the freezing tube, and promote the evaporation of water. In this way, the soil is frozen, and the frozen soil is formed. The frozen soil has the characteristics of high strength, good sealing and so on. This is the so-called freezing method ${ }^{[1]}$. The criterion for judging the performance of this method is the formation of the temperature, as to whether or not the normal operation of the temperature is determined by the temperature of the brine. But in the practical application, it is found that the observation of the frozen wall cannot be directly observed, and it is needed monitoring system to monitor effectively, The main task of the monitoring system is to make the staff to adjust and predict the development of the frozen wall in time by monitoring the data.

In recent years, along with the monitoring system has been concerned, more and more new monitoring system is rising. For example, constantan thermocouple line measurement, distributed optical fiber temperature measurement system and 1-wire bus technology to design the monitoring system. In this paper, the one line bus technology is applied to develop the mine ground freezing monitoring system, in the actual application, the one line bus is relatively simple, you can arbitrarily set the measurement point that affect the total length cannot be too long, which caused the number of monitoring points is limited, so it is not widely promoted in practice. In this paper, we design a set of freezing monitoring system combined with the one line bus technology, combined with the real coal mine for real time monitoring.

\section{One line bus technology}

\subsection{One line bus network structure}

The so-called one line bus is through a bus cable to connect the bus management unit with the line bus protocol sensors, data transmission and signal transmission are implemented. It has the characteristics of multi layer overlapping and uniqueness. Because there is only one bus, it makes the cost of communication line number are reduced largely, and the cost consumption is reduced greatly. At the same time, it also can make the circuit more simply, and it is favorable to the maintenance and wiring. Due to its simple circuit, the reliability is greatly enhanced, to realize the possibility of a line to achieve of two-way data transmission. Taking into account these advantages, it has obtained more and more attention and concern in the field of engineering testing, which can meet the application requirements ${ }^{[3-5]}$. 
The basic structure of one line bus monitoring network is shown in Figure 1, in the following Figure 1, we can see the basic structure of the one line bus monitoring network. The whole structure can be seen that the bus unit is connected with the one line bus cable, and the one line bus cable is connected with a lot of one line bus devices. The one line bus device is the slave structure of the bus unit, which is subject to the management and control operation status of the bus unit, and at the same time, the data can be obtained and upload back to the bus unit. And this kind of structure is very strict. The so-called strict refers to the one line bus devices in the operation of the unit not able to run separately without the management unit. Only when the bus management unit is started, the one line bus device is able to start and communicate.

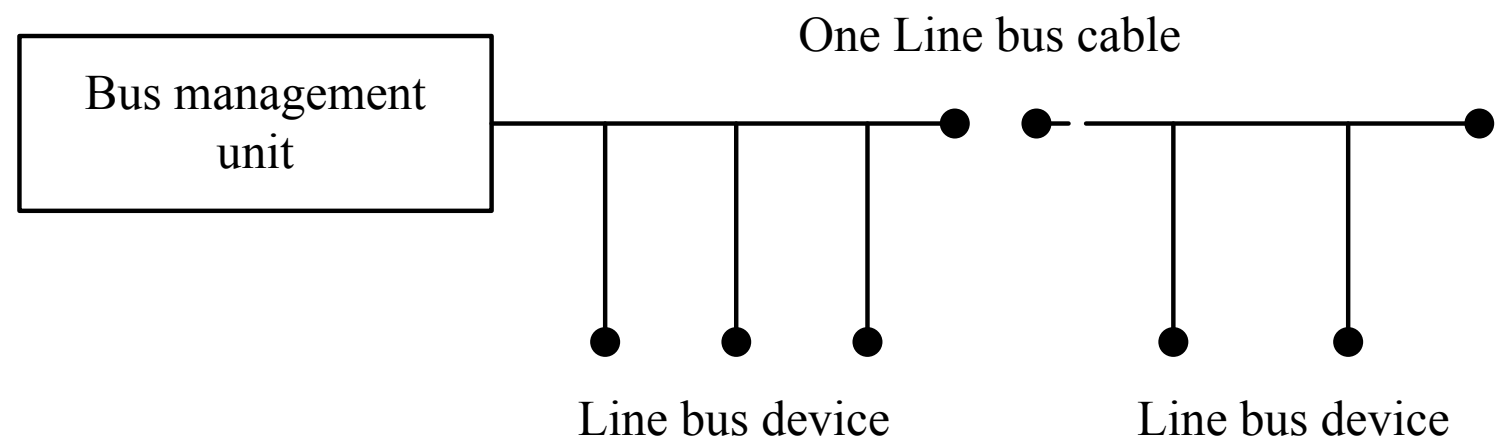

Fig. 1 Basic structure of one line bus monitoring network

Along with the continuous development of the one line bus technology, it has obtained more and more attention and wide application, and the line bus device connected with the bus unit is constantly growing and becoming more and more multiply. For example, we know that DS18B20, which is a digital temperature sensor. This kind of sensor is an one line bus device which is developed by the one line bus technology. Because of its many advantages, it has been widely used in environmental monitoring, freezing monitoring and other monitoring projects for real-time temperature monitoring.

\subsection{Key technology of one line bus}

As expressed above, the one line bus technology is simple, but at the same time, the transmission distance cannot be too long, which caused working range of the bus technology will be affected by the transmission distance from the bus. In addition, the one line bus device is provided by the component supplier, which makes performance of the one line bus device of one line bus monitoring network is also very important for the whole system. Due to the one line bus structure is a series structure, so that when a one line bus device appeared in the case of a short circuit, so that the entire network may fall into paralysis resulted from its impact. Therefore, the solution to this kind of situation has become the important problem of the one line bus technology subject.

\section{Hardware design of system}

\subsection{System structure}

The system structure of the formation freezing monitoring is shown in Figure 2. As can be seen from the figure, the whole system is connected with the monitoring module through the one line bus network, which is the core of the whole system ${ }^{[3]}$. Line bus devices and cells pressure sensor, temperature sensor, sensor and flow meter are connected together, and the monitoring module is connected with the monitoring computer. The monitoring computer can be found through the cable connection number of radio stations, these stations and monitoring modules are equipped with wireless signal transmission tower, o facilitate the wireless connection between the devices. Previously we had said that the data of data cannot be infinitely increased, because each monitor module has an address, and the address is unique, so that the most monitoring modules can reach 256 of one line bus network. 


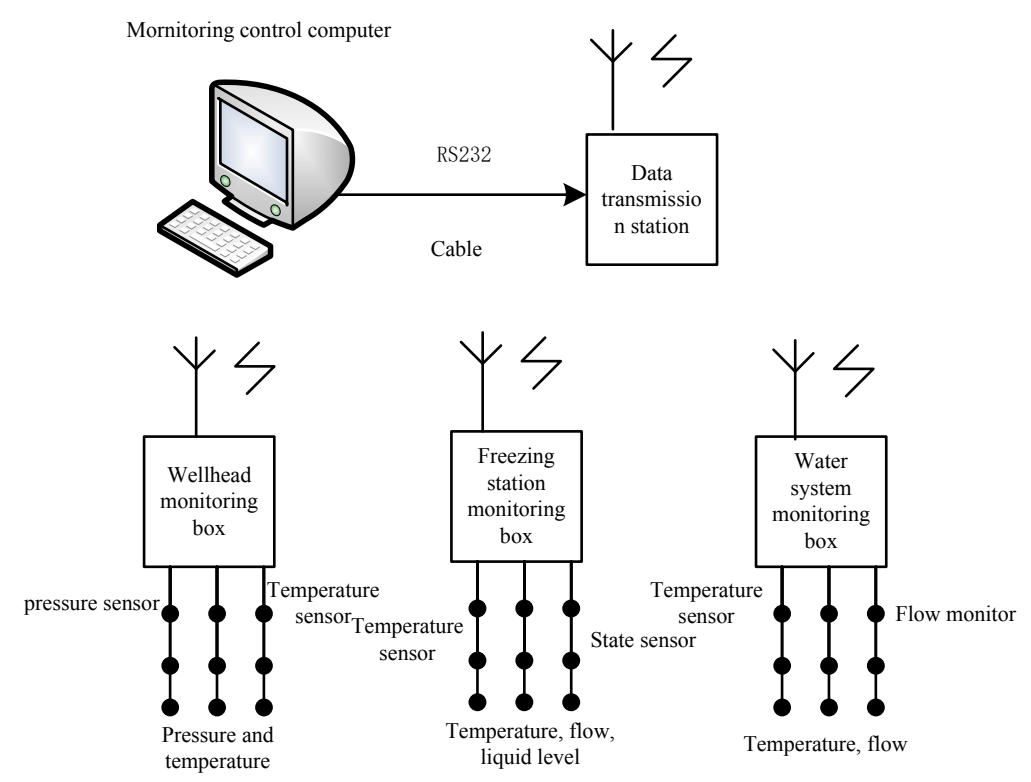

\subsection{Monitoring of mixing parameters}

Fig.2 Formation of ground freezing monitoring system

In the actual detection of the underlying frozen, there are not only the temperature signal needs to be detected, often there are many other signals need to be monitored, but the temperature signal is the main monitoring indicators. For other monitoring signals, such as pressure signals, flow signals, etc., they can be collected by these sensors and connected to a dedicated line of bus devices. Finally, through the device, the data and signal monitoring is achieved, and the data are transmitted to the monitoring system.

\section{Software design of the system}

Software main interface of ground freezing monitoring system is shown in Figure 3, we can see the main interface of the software is designed effectively, the real time monitoring system is developed, and it is mainly used to test temperature, of course, other parameters can be tested with monitoring. Based on this system software interface, the software can realize the temperature display, humidity display, real-time monitoring reports and historical curves and other functions, but mainly to carry out the test results display. As the above function, combined with figure 4, carry out the parameter setting, the data acquisition configuration interface is shown in Figure 4.

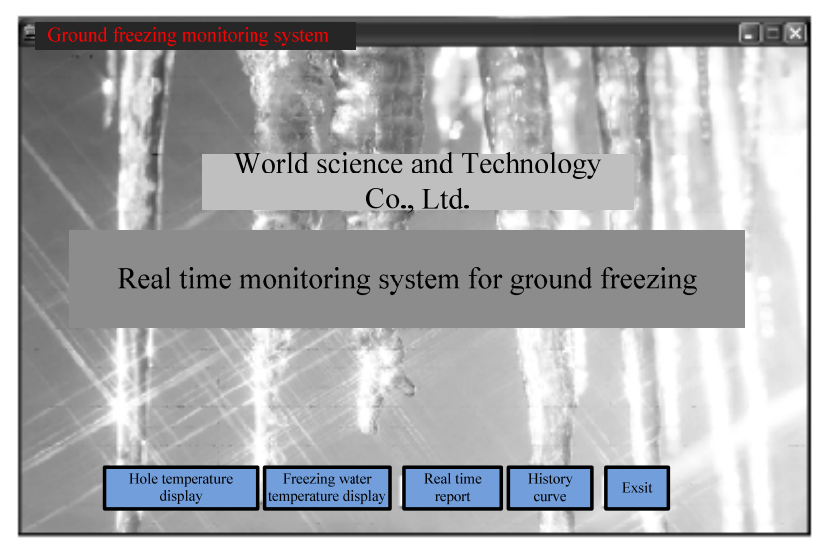

Fig. 3 Software main interface of ground freezing monitoring system 


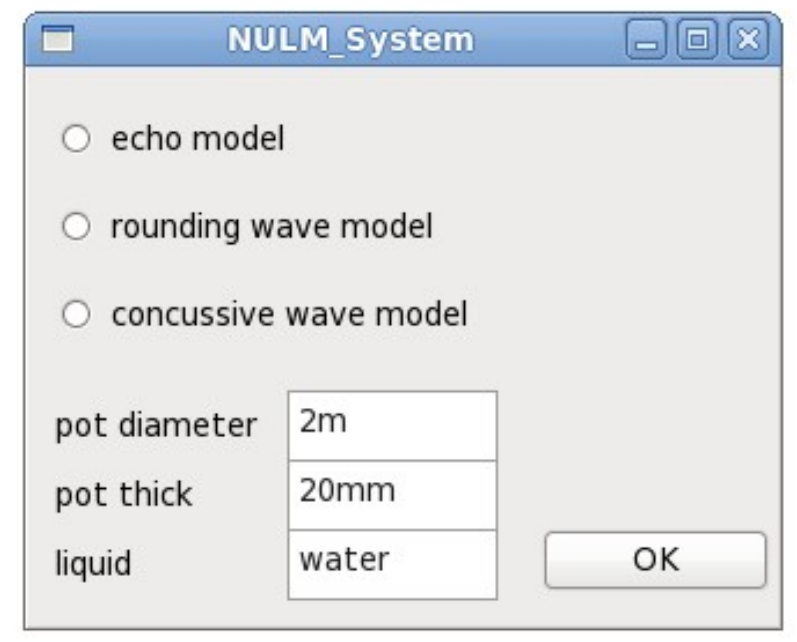

Fig.4 Data acquisition configuration interface

\section{System applications}

The frozen bus line monitoring system is designed in this paper, and combined with Zhangji coal mine auxiliary shaft freezing engineering examples for practical application. Some parameters of temperature measuring points and temperature measuring holes in the system are presented as: thermometer holes number is 4 , and there are total of 99 points. Total of temperature measurement points is 243 . For the test data, through the transformation of digital signals, the temperature of each measurement point will be displayed through the software main interface. See the history curve of the main interface this option, click on this option, you can display the temperature curve of salt water in the main interface. As shown in Figure 5, the further analysis can be obtained, and the status of the operation can be monitored.

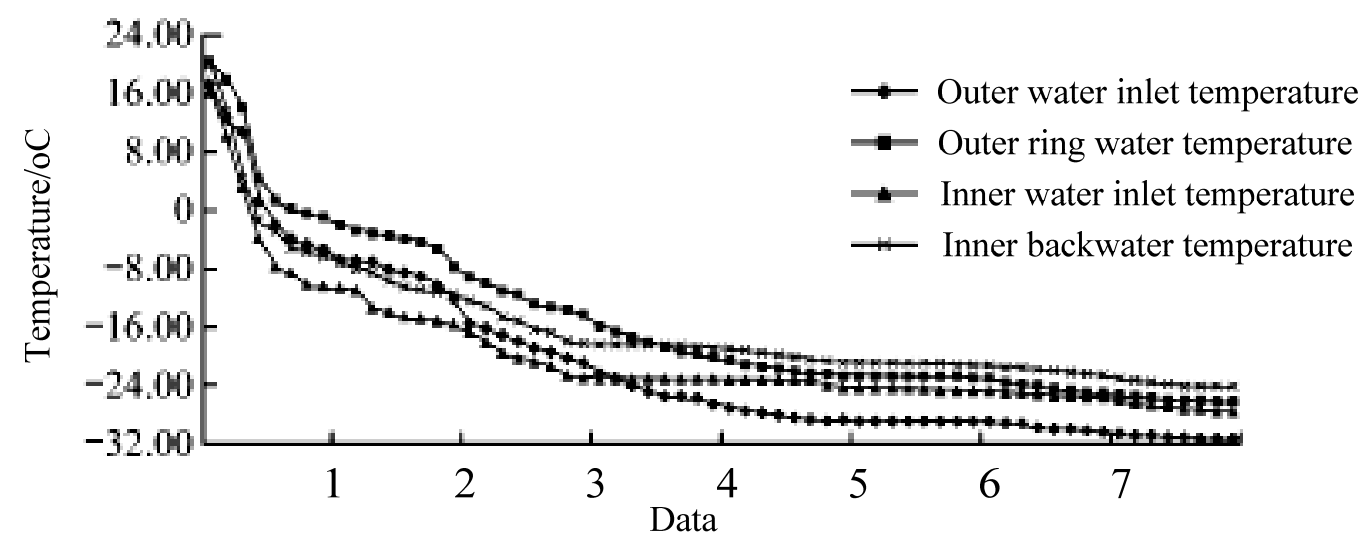

Fig.5 Temperature curve of brine dry pipe 


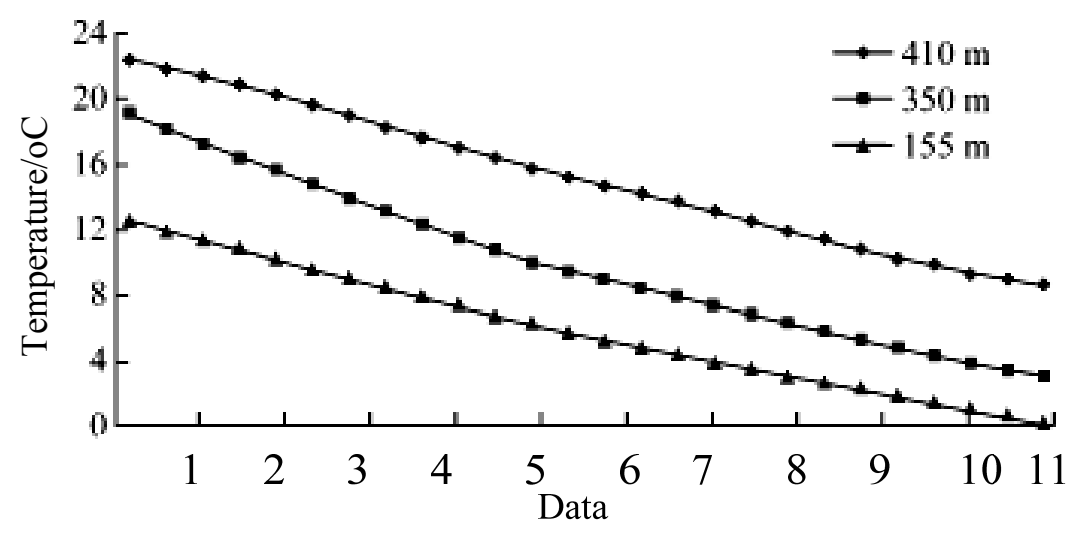

Fig. 6 Temperature curve of a temperature measuring hole in gravel layer

The temperature curve of a temperature measuring hole in gravel layer is shown in Figure 6. If the temperature data of each temperature measurement hole is obtained by analysis in advance, the system can be stored in the database to be established in the future. Of course, this system is very thoughtful consideration, in order to make the analysis more comprehensive, for the monitoring of the flow factor in the freezing monitoring is also included in the scope of monitoring. The flow monitoring curve is shown in Figure 7.

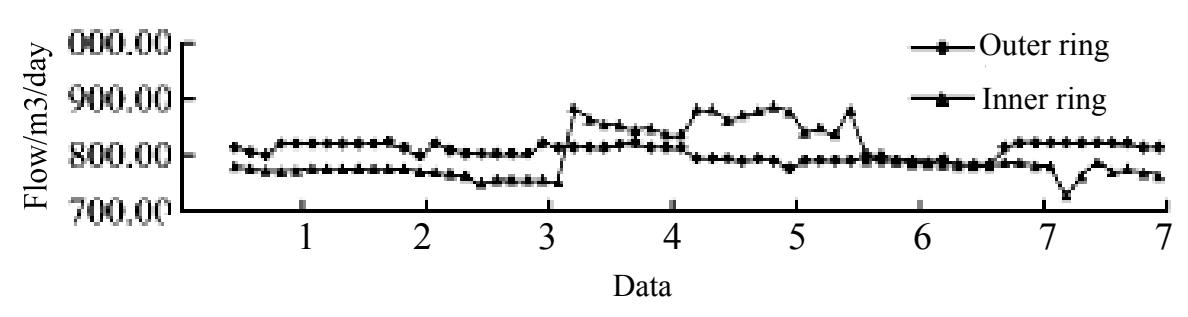

Fig. 7 Flow monitoring curve

\section{Conclusions}

In this paper, the one line bus monitoring system of mine stratum freezing is developed, it can provide reliable data and graphical analysis curve for the theoretical analysis. Combined with the Zhangji coal mine auxiliary shaft freezing engineering example, the stability and the accuracy of the verification and inspection are proved, that it has excellent performance in monitoring. However, due to the performance of the system is still not perfect enough, it needs further improvement in the future. Due to the limitation of the length of the bus, the tendency of the mining coal seam is getting deeper and deeper, and the future development trend is advancing towards the development of distributed optical fiber type.

\section{References}

[1] Zhang Yi, Zhou bingying, Hu Guangbo. Hardware System Design of Underwater Motor Pump Faults Diagnose Detector[J].Computer \& Digital Engineering,2012,40(11): 162-166.

[2] JIA Zheng. Accounting software-based design methodology and application of ERP[J]. Electronic Design Engineering, 2014 (17) :56-58.

[3] Shao-lei.Wireless intelligent lighting control system based on Zigbee technology[J].SAMSON,2015(19):125-126. 
[4] Chen Rongjiang, Yao Jianhui xiang-long meng,nc hydraulic station of coal mine based on the chip control,the design and simulation [J].Science and technology,2012,28(8):103-106.

[5] WANG Huixian, JIN Huijia, WANG Jiaolong, JIANG Wanshou. Optimization Approach for Multi-scale Segmentation of Remotely Sensed Imagery under k-means Clustering Guidance[J]. Acta Geodaetica et Cartographica Sinica, 2015, 44(5): 526-532. 